\title{
Mechanism of Bacteriophage Lytic Enzyme in Phage Therapy against Streptococcal Infection by in Silico Approach
}

\author{
Article by Chethan Kumar $S^{1}$, VinodKumar C.S ${ }^{2}$ \\ ${ }^{1}$ Research Coordinator, S. S. Institute of Medical Sciences and Research Centre, India \\ ${ }^{2}$ Professor, Department of Microbiology, S. S. Institute of Medical Sciences and Research \\ Centre, India \\ E-mail: chethan_kumar@outlook.com
}

\begin{abstract}
Lysins or Lytic enzymes of Bacteriophage are highly evolved molecules produced to release their progeny by hydrolyzing the bacterial host cell wall. Now days, due to Multi Drug Resistance in many streptococcal infections; this mechanism is exploited as an alternative therapy against traditional antibiotic therapy. But in the treatment of phage therapy the mode of action is still unclear in the literatures. This study will evidence the probable mode of mechanism by the lysin in the breakage of host cell wall by In Silico approach. 3-D structure of Lysin was retrieved from Protein Data Bank and structure of peptidoglycan is retrieved from the PubChem. Docking studies was performed using Hex 6.3 taking lysin as receptor and peptidoglycan as ligand. Results were visualized in PyMol molecular visualization software. Docking studies showed the hydrophilic interaction between the peptidoglycan and lysin. The interacting residue of lysin belongs to CHAP domain which is responsible for the amidase catalytic activity which results in the breakage of cell wall for the release of their progeny. Molecular interactions between the lysin and peptidoglycan showed the possible mechanism for lysin which is responsible for breaking the major bonds in peptidoglycan layer for the release of bacteriophage progeny inside the host bacterial cell. This study will give the further evidence for the mode of action and by understanding this mechanism further improved therapies can be achieved in the Multi Drug Resistance bacterial infections.
\end{abstract}

Keywords: Bacteriophage, Streptococcus spp, Multi Drug Resistance bacterial infections, Lysin, Peptidoglycan, Docking studies.

\section{Introduction}

With the rising prevalence of antibiotic-resistant bacteria ${ }^{[1,2]}$ alternatives to treatment with antibiotics are receiving increased attention. One such alternative is the possible therapeutic use of bacteriophagesviruses that parasitize and kill bacteria. When first discovered early in the previous century, the potential to use phage against pathogenic bacteria to cure infections, so-called "phage therapy", was immediately grasped, but the emergence of antibiotics put this goal on the back burner ${ }^{[3,4]}$. However, as multi-drug resistant bacteria have become an enormous public health problem, there is a renewed interest in phage therapy. At our centre, bacteriophages for MDR Staphylococcus aureus, Pseudomonas aeruginosa, Klebsiella pneumonia, Acinetobacter baumannii, Enterococci species, E.coli and Streptococcus species has been isolated and utility of these phages to rescue wound infections in animal model has been studied [5-10].

Bacteriophage typically encodes holins and lysins as part of their lytic system to achieve virus exit from the host bacterial cell ${ }^{[11]}$. Holins are responsible for forming pores in the cytoplasmic membrane, following which lysins, having accumulated in the cytoplasm, are responsible for degradation of the peptidoglycan layer i.e., cell wall. Damage to this layer results in rapid cell rupture and concomitant virus release through loss of osmotic integrity. So lysins play a very important role in the lysis of bacterial cell 
wall that results the potential therapeutic effect against multi drug resistant bacteria in foot infections of type II diabetes mellitus.

Lysins derived from phage that infect Gram-positive bacteria are generally composed of a single polypeptide consisting of an $\mathrm{N}$-terminal catalytic domain and a $\mathrm{C}$-terminal cell wall binding domain (CBD) held together by a short flexible linker ${ }^{[12]}$. In some rare cases, two or three catalytic domains may be linked to a single binding domain ${ }^{[13,14]}$. With few exceptions, the catalytic domain is usually represented by one of four families of peptidoglycan hydrolases: $\mathrm{N}$-acetylglucosaminidases, $\mathrm{N}$ acetylmuramidases (lysozymes), $\mathrm{N}$-acetylmuramoyl-L-alanine amidases and endopepridases ${ }^{[15]}$.

The Streptococcal C1 phage lysine, PlyC, is the most potent lysine described to date. PlyC is furthermore unique among the Gram-positive lysins in that it consists of two separate proteins-a single 50-kDa PlyCA subunit that is suggested to form a complex with at least eight 8-kDa PlyCB subunits ${ }^{[16]}$. PlyCA is known to contain an active cysteine-histidine dependent amino hydrolases/peptidase (CHAP) domain, a fold distantly related to the papain-like cysteine-protease family, with Cys 333 and His 429 shown to be essential for amidase catalytic activity ${ }^{[16]}$. The PlyCB octamer is suggested to represent the CBD because purified material lacking the PlyCA subunit was able to specifically bind Streptococcus pyogenes, S.ubris, S.equi, and groups C and E Streptococci, but not other bacterial species (i,e., Streptococcus agalactiae, and Streptococcus mutans) ${ }^{[16]}$.

\section{Materials and methods}

The bacteriophage for Streptococcus pyogenes was isolated from different sources of water by the method of Smith and Huggins ${ }^{[17]}$. In vitro confirmation of bacteriophage activity was done on bacterial lawn, prepared on nutrient agar plates employing $1.0 \mathrm{ml}$ of $24 \mathrm{hr}$ culture by flooding and draining out the excess. Wells were dug into the agar by employing a sterile cork borer and the $20 \mu$ phage suspension were loaded into each of the well. Sterile distilled water served as the control. The plates were incubated at $37^{0} \mathrm{C}$ for $24 \mathrm{hr}$. There after the zone of inhibition, if any, was recorded. The plaques if obtained were further passaged on the same target bacterial host and other members of the same genus to reconfirm its diversified activity ${ }^{[8]}$.

\section{Computational methods}

To understand the replication process of bacteriophage, an In Silico method is approached to provide more evidence that how this will be achieved inside the host cell. The experimentally solved 3Dimensional structure of Lysin is retrieved from the Protein Data Bank (http://www.rcsb.org/pdb/home/home.do) and Peptidoglycan is retrieved from the PubChem (http://pubchem.ncbi.nlm.nih.gov/). Peptidoglycan sdf extension file retrieved from the PubChem is converted into pdb using Open Babel software. Docking of Lysin and Peptidoglycan is performed using Hex 6.3 software and obtained result is visualized in PyMol software.

\section{Results}

Streptococcus phage isolated formed plaques on $88 \%$ of Streptococcus species isolated from diabetic foot infection specimens. The 3-Dimensional structure of Lysin was retrieved from Protein Data Bank bearing the PDB ID: 4F88 (Table 1, Fig 1), consists of 465 amino acid residues and Peptidoglycan was retrieved from PubChem bearing the accession number of CID 9816401 (Fig 1). The retrieved Lysin file consists of two complete PlyC molecules. One molecule was removed using PyMol software to perform docking studies. Docking studies revealed that oxygen atom of Peptidoglycan is showing hydrophilic interaction with hydrogen atom of Valine 388 in Lysin (Fig 2).

\section{Discussion}

The discovery of antibiotics was a leap in modern medicine. They have been able to stop the growth or kill many different kinds of microorganisms. However, bacteria have proven to be much more innovative and adaptive than we imagined and have developed resistance to antibiotics at an ever-increasing pace. 
Bad practices and mismanagement have only exacerbated the situation. We could soon return to a state of medical health that was as dire as that which occurred prior to antibiotic use. The situation has reached such a decisive point that the World Health Organization (WHO) has cautioned of a return to a "preantibiotic era". The bacteriophages used in animal experiments have proved that they can resolve wound infection effectively than antibiotics ${ }^{[6-10]}$

Docking study on phage and the host revealed that PlyC consists of two components. A catalytic subunit or domain called PlyCA and binding subunit or domain called PlyCB. The binding domain is responsible for binding in peptidoglycan layer whereas catalytic subunit is responsible for breakage of major bonds in peptidoglycan layer, results in bacterial lysis.

In PlyCA there are two distinct catalytic domains to achieve cell lysis. One is CHAP (CysteineHistidine-dependent Aminohydrolase/Peptidase) domain, residue ranges from 309 to 465 amino acids, responsible for amidase catalytic activity and second one is GyH (Glycosyl hydrolase) domain, residue ranges from 1 to 205 amino acids, responsible for glycosidase activity.

From this result, we can understand that the interaction showing amino acid residue Valine 388 lies in the CHAP domain and this domain is responsible for the amidase catalytic activity. Due to this catalytic activity, the breakage of major carbon-nitrogen bonds of the Peptidoglycan in the cell wall will occur. This results in the degradation of bacterial cell wall and facilitates virus egress from the host. This is how we can predict the bacterial infections are countered in the Streptococcal infections. The studies on utility bacteriophages reinforces that the phages could be used in a situations where there is no substitutes available for treating multi-resistant strains or as a valuable adjunct to antibiotics when the bacteria are still susceptible.

\section{Conclusion}

Molecular interactions between the lysin and peptidoglycan showed the possible mechanism for lysin which is responsible for breaking the major bonds in peptidoglycan layer for the release of bacteriophage progeny inside the host bacterial cell. This study will give the further evidence for the mode of action and by understanding this mechanism further improved therapies can be achieved in the Multi Drug Resistance bacterial infections.

\section{Tables and figures}

Table 1. Retrieved 3-D structure of Lysin form PDB

\begin{tabular}{|l|l|l|l|}
\hline PDB ID & Organism & Sequence ID & Amino acid residues \\
\hline 4F88 & Streptococcus phage C1 & Q7Y3F1 & 465 \\
\hline
\end{tabular}


DOI: $10.21522 /$ TIJCR.2014.04.02.Art009

ISSN: $2520-3096$

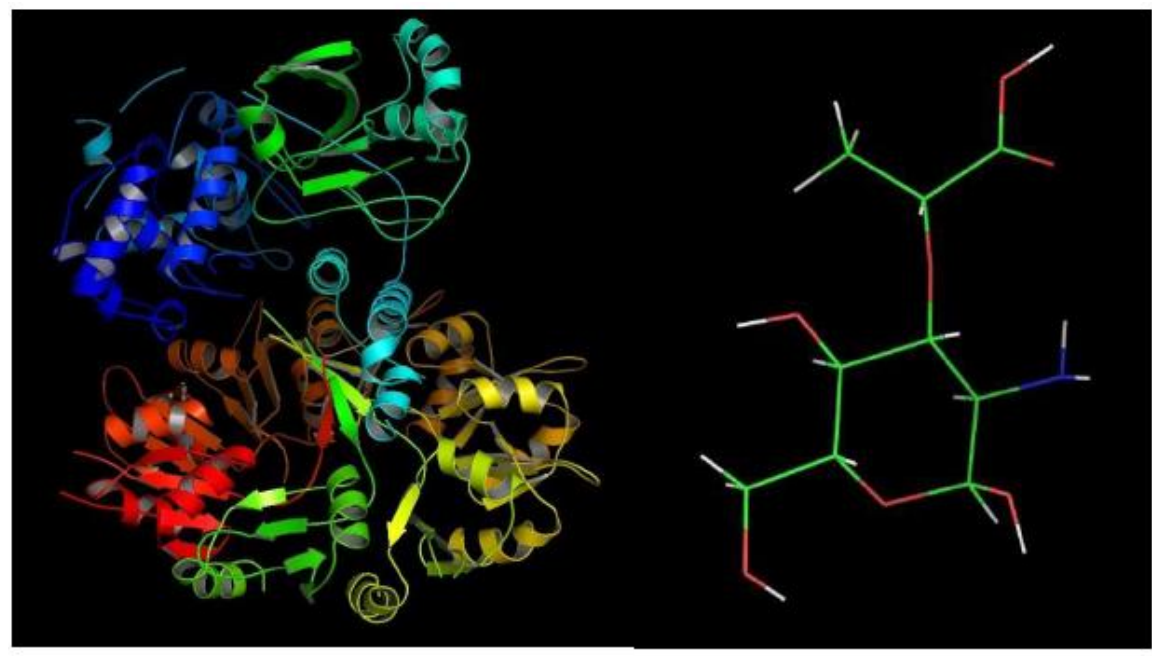

Figure 1: Three-Dimensional structure of Lysin and Peptidoglycan

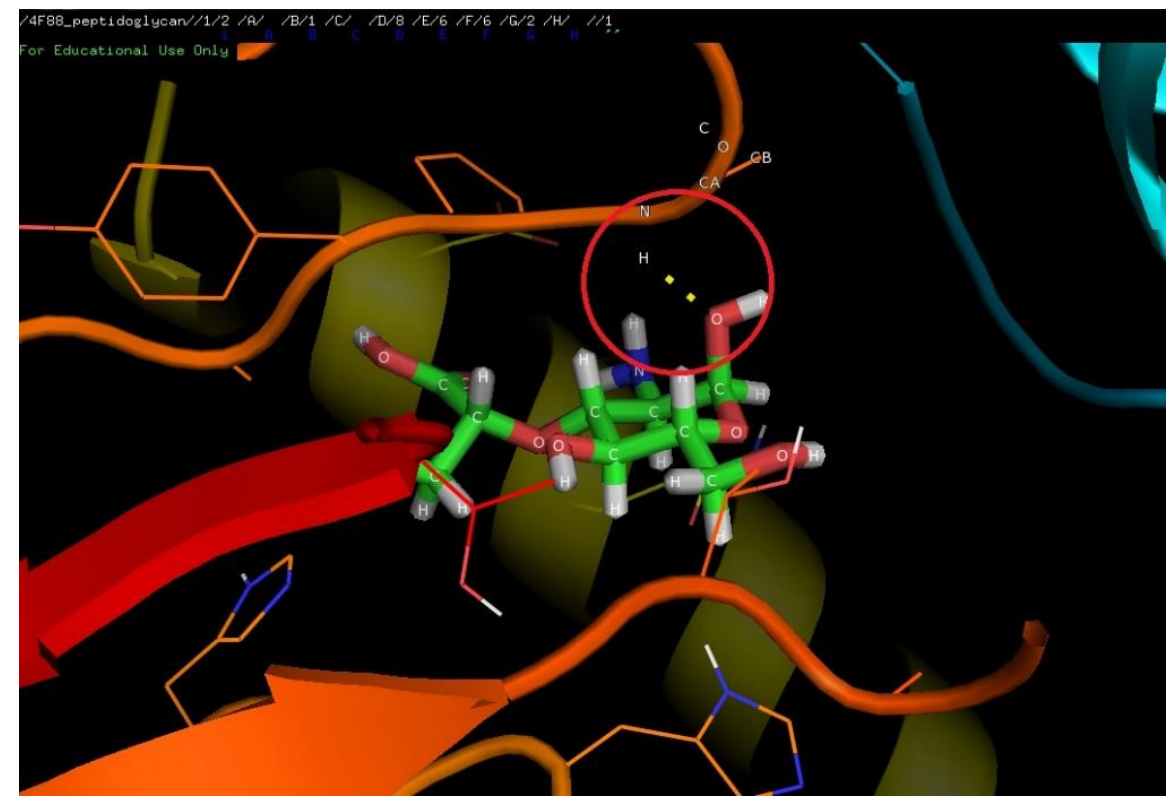

(a) 


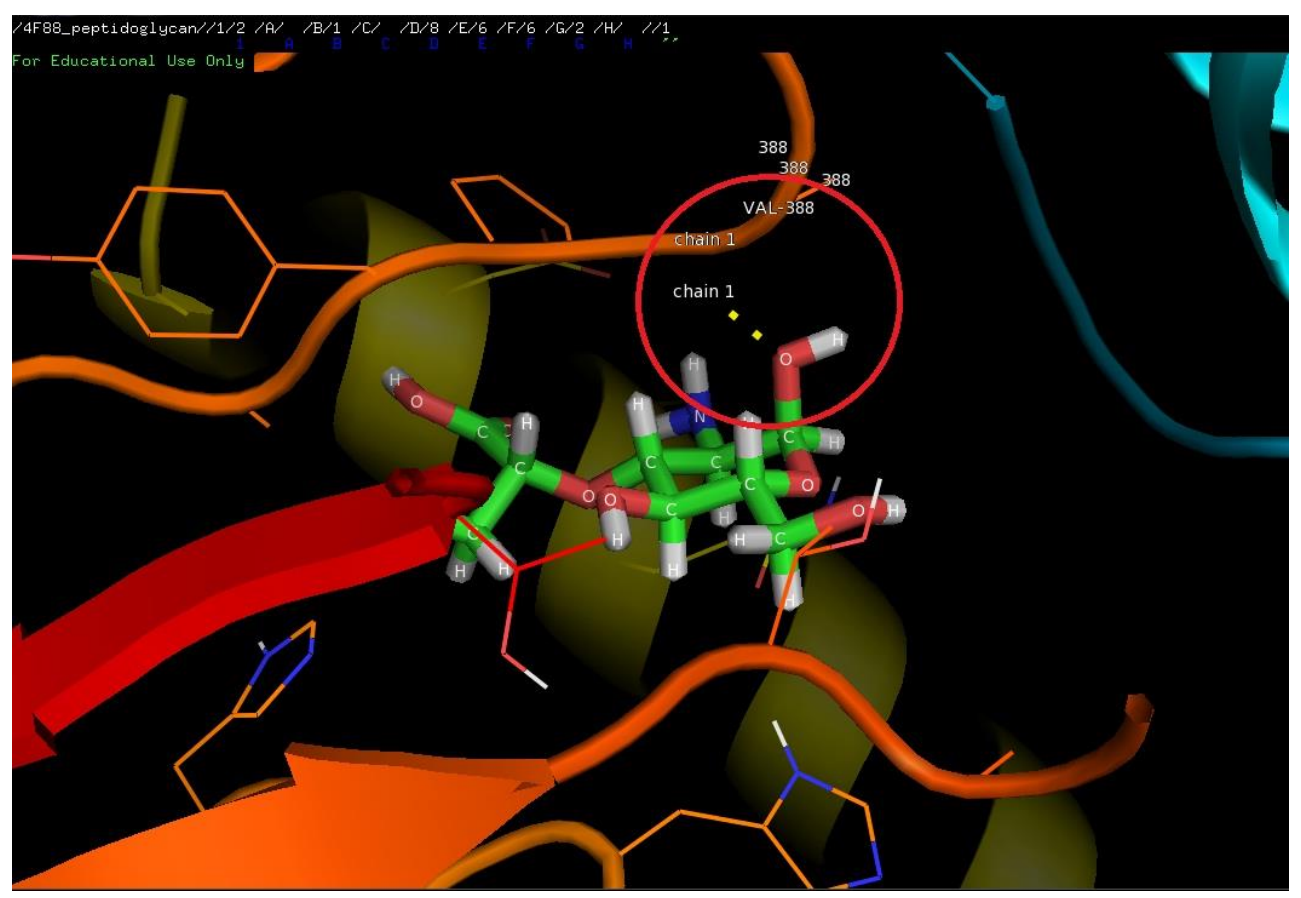

(b)

Figure 2. (a) Docking results of Lysin and Peptidoglycan, (b) Interaction between Hydrogen atom of Valine 388 and Oxygen atom of Peptidoglycan in cartoon representation

\section{References}

[1]. Berkowitz FE. Antibiotic resistance in bacteria. South Med J 1995;88:797-804.

[2]. B. Levin, J. Bull. Population and evolutionary dynamics of phage therapy. Nature Reviews Microbiology 2004; 2: 166-173.

[3]. Hermoso JA, Garcia JL, Garcia P. Taking aim on bacterial pathogens: From phage therapy to enzybiotics. Curr Opin Microbiol 2007; 10:461-472.

[4]. Navarre WW, Ton-That H, Faull KF, Schneewind O. Multiple enzymatic activities of the murein hydrolase from staphylococcal phage phi11. Identification of a D-alanyl -glycine endopeptidase activity. J Biol chem 1999; 274:15847-56.

[5]. Nelson D, Schuch R, Chahales P, Zhu S, Fischetti VA . PlyC: A multimeric bacteriophage lysine. Proc Natl Acad Sci USA 2006; 103:10765-70.

[6]. Pritchard DG, Dong S, Baker JR, Engler JA. The bifunctional peptidoglycan lysine of Streptococcus agalactiae bacteriophage B30. Microbiology 2004; 150:2079-87.

[7]. Stark CJ, Hoopes JT, Bonocora RP, Nelson DC. Bacteriophage lytic enzymes as antimicrobials. Bacteriophage in the Detection and control of Foodborne Pathogens, eds Sabour PV, Griffith MW (ASM Press, Washington DC), 2010. pp 137-156.

[8]. Smith H W, Huggins M B. Successful treatment of experimental E.coli infections in mice using phage; its superiority over antibiotics. J Gen Microbio. 1982; 128:307-318.

[9]. S. Matsuzaki, M. Rashel, J. Uchiyama, S. Sakurai, T. Ujihara, M. Kuroda, M. Ikeuchi,T. Tani, M. Fujieda, H.Wakiguchi, S. Imai, Bacteriophage therapy: a revitalized therapy against bacterial infectious diseases. J. Infect. Chemother. 2005; 11: 211-219.

[10]. Tenover FC, Hughes JM. The challenges of emerging infectious diseases: development and spread of multiply resistant bacterial pathogens. JAMA 1996; 275:300-4.

[11]. VinodKumar C. S, Suneeta Kalsurmath, Neelagund Y.F. Utility of lytic bacteriophage in the treatment of multidrug resistant Pseudomonas aeruginosa septicemia in mice. Ind J Patho Microbiol 2008;51(3):360-366 
DOI: $10.21522 / \mathrm{TIJCR} .2014 .04 .02 . A r t 009$

ISSN: $2520-3096$

[12]. VinodKumar C. S, Suneeta Kalsurmath, Neelagund Y.F. Bacteriophage in the treatment of experimental septicemic mice from a clinical isolate of multidrug resistant Klebsiella pneumoniae. J Commun Dis. 2005;37(3): 749-753

[13]. VinodKumar C. S, Srinivasa H, Basavarajappa K.G, Nitin Bandekar. Isolation of Bacteriophages for MRSA obtained from Diabetic foot- A Possible treatment Option in infections. Inter J Biotechnol Biochem. 2010; 6(5): 801809.

[14]. VinodKumar C. S, Srinivasa H, Basavarajappa K.G, Geethalakshmi S, Nitin Bandekar. Isolation of Bacteriophages to MDR Enterococci obtained from Diabetic foot- A Novel antimicrobial agent waiting in the Shelf?. Ind J Pathol Microbiol. 2011;54(1):90-96

[15]. VinodKumar C. S, Srinivasa H, Basavarajappa K.G, Umakanth Patil, Nitin Bandekar, Rajashri Patil. Abrogation of Staphylococcus aureus wound infection by bacteriophage in diabetic rats. International journal of pharmacy and drug research. 2011:3(3); 202-207

[16]. VinodKumar C. S, Srinivasa H, Basavarajappa K.G, Umakanth Patil, Nitin Bandekar, Rajashri Patil. Effectiveness of bacteriophage in the treatment of Staphylococcus aureus wound infection in the diabetic animal model. Asian J Pharm Res. 2012;5: 123-127

[17]. Young R. Bacteriophage lysis: Mechanism and regulation. Microbial Rev 1992; 56:430-481. 